ANRAD

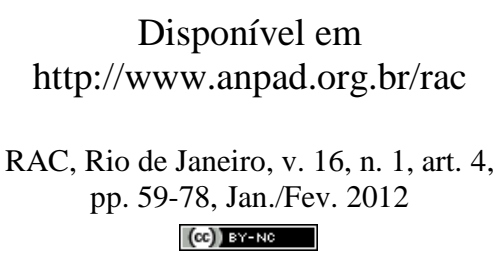

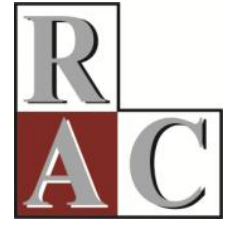

\title{
Intervenientes na Transferência de Tecnologia Universidade- Empresa: o Caso PUCRS
}

\section{Factors that Influence the University-Industry Technology Transfer Process: the Case of PUCRS}

\author{
Lisiane Closs * \\ E-mail: 1closs@terra.com.br \\ Pontifícia Universidade Católica do Rio Grande do Sul - PPGAd/PUCRS \\ Porto Alegre, RS, Brasil. \\ Gabriela Ferreira \\ E-mail: gcferreira@pucrs.br \\ Pontifícia Universidade Católica do Rio Grande do Sul - PPGAd/PUCRS \\ Porto Alegre, RS, Brasil. \\ Cláudio Sampaio \\ E-mail: csampaio@pucrs.br \\ Pontifícia Universidade Católica do Rio Grande do Sul - PPGAd/PUCRS \\ Porto Alegre, RS, Brasil. \\ Marcelo Perin \\ E-mail: mperin@pucrs.br \\ Pontifícia Universidade Católica do Rio Grande do Sul - PPGAd/PUCRS \\ Porto Alegre, RS, Brasil.
}

* Endereço: Lisiane Closs

Av. Ipiranga, 6681, prédio 50, sala 1105, Porto Alegre/RS, 90619-900.

Copyright (C) 2012 RAC. Todos os direitos, até mesmo de tradução, são reservados. É permitido citar parte de artigos sem autorização prévia, desde que seja identificada a fonte. 


\title{
Resumo
}

Poucos estudos abordam a transferência de tecnologia universidade-empresa (TTUE) em países em desenvolvimento (Póvoa \& Rapini, 2010), sobretudo, no Brasil, em universidades privadas (Closs \& Ferreira, 2010). Este trabalho, nesse sentido, busca identificar e analisar intervenientes em processos envolvendo patentes acadêmicas, investigando pesquisadores e a gestora do Escritório de Transferência de Tecnologia (ETT), no estudo do caso da PUCRS. Muitos dos intervenientes assemelharam-se aos identificados por outros estudos nacionais e internacionais. Entre os motivadores que se destacaram estão os desafios científicos e a competição para produzir patentes. O ambiente de inovação foi favorecido por políticas institucionais e o acesso à informação, estruturas de laboratórios, equipes qualificadas e grupos de pesquisa multidisciplinares viabilizaram a geração de inventos. A existência do parque tecnológico na universidade facilitou a disseminação do conhecimento tácito entre a universidade e empresas. A dificuldade para conciliar pesquisa, patenteamento e docência requer atenção, pois os pesquisadores foram essenciais para o sucesso na comercialização de tecnologias acadêmicas. Sugere-se, desse modo, a criação de políticas que possibilitem mais tempo aos pesquisadores para a atuarem na Transferência de Tecnologia Universidade-Empresa (TTUE) e o desenvolvimento de suas habilidades relacionais e comerciais.

Palavras-chave: transferência de tecnologia; universidade-empresa; patentes.

\begin{abstract}
Few studies have addressed the university-industry technology transfer (UITT) process in developing countries (Póvoa \& Rapini, 2010); especially, in Brazilian private universities (Closs \& Ferreira, 2010). This paper seeks to identify and analyze elements in processes involving academic patents through a case study investigating researchers and the TTO manager of PUCRS. Many results were similar to those identified by other national and international studies. Among the drivers that stand out are scientific challenges and competition to produce patents. An innovative environment was favored by institutional policies. Access to information structures, laboratories, skilled staff and multidisciplinary research groups favored invention. The existence of the technology park in the university also facilitated the dissemination of tacit knowledge between universities and companies. Reconciling research, teaching, and patenting with each other is an issue that requires special attention, because researchers were essential to successful commercialization of academic technology. We suggest the creation of policies which allow researchers more time for UITT and developing their interpersonal and commercial skills.
\end{abstract}

Key words: technology transfer; university-industry; patents. 


\section{Introdução}

A transferência de tecnologia universidade-empresa (TTUE) vem adquirindo importância estratégica em muitos países, pois representa fonte de recursos para a pesquisa acadêmica, inovação para as empresas e desenvolvimento econômico para os governos (Muscio, 2010). Pioneiro no desenvolvimento de mecanismos para favorecer esse processo, o governo dos Estados Unidos (EUA) estabeleceu os direitos de propriedade intelectual (PI) sobre resultados de inventos financiados por fundos federais para universidades, em 1980, através do Bayh-Dole Act, permitindo seu patenteamento e estimulando sua comercialização.

Buscando reproduzir o crescimento de patentes resultante, aparentemente, dessa lei (Golish, Besterfield-Sacre, \& Shuman, 2008; Sampat, 2006), diversos países europeus modificaram suas legislações sobre PI (OECD, 2003). Por sua vez, o Brasil instituiu, em 1996, a Lei de Propriedade Intelectual, que possibilitou a proteção tecnológica em áreas onde a pesquisa acadêmica do país era forte, aumentando o interesse em patentes. A Lei de Inovação (Lei Federal n. ${ }^{\circ} 10.973$, 2004) permitiu parcerias entre instituições de pesquisa, universidades e firmas, buscando reforçar suas relações e incentivar a inovação, com isso, representando o mecanismo legal do governo para aumentar o número de registros de patentes no país (Corrêa, 2007).

Mudanças em legislação isoladamente, no entanto, não resultaram em crescimento expressivo de patentes em muitos países (Baldini, 2006; Sampat, 2006). No caso dos EUA, características do seu sistema universitário, o aumento de financiamentos de pesquisas acadêmicas pela indústria e o auxílio crescente dos escritórios de transferência de tecnologia (ETT) (Azagra-Caro, Carayol, \& Llerena, 2006) foram também essenciais para o seu desempenho em geração de patentes universitárias. A análise da TTUE, por conseguinte, deve integrar a compreensão de seu contexto de ocorrência, pois diferenças institucionais e organizacionais interferem decisivamente nesse processo (Baldini, 2006).

No caso do Brasil, as empresas, em geral, prescindem de uma cultura inovadora (Santos \& Solleiro, 2006) e as universidades assumem papel de destaque na geração de patentes. Em levantamento realizado pelo Instituto Nacional da Propriedade Industrial (INPI), entre 2000 e 2005, quatro universidades estavam entre os vinte maiores depositantes de patentes nacionais (Póvoa \& Rapini, 2010), evidenciando esta questão. Nesse contexto, o maior número de pesquisadores doutores e mudanças em sua postura com relação ao patenteamento de inventos foram determinantes para o crescimento intenso do número de depósitos de patentes ocorrido no país, a partir de 2002 (Póvoa, 2008).

Apesar da relevância da TTUE para o desenvolvimento econômico das nações, poucos estudos foram realizados sobre o tema, em países em desenvolvimento (Póvoa \& Rapini, 2010). No Brasil, sobretudo, há uma lacuna de trabalhos que investiguem universidades privadas (Closs \& Ferreira, 2010). Há, ainda, uma carência de insights advinda da investigação de universidades individuais e de mecanismos que favoreçam o patenteamento acadêmico em âmbito micro (Azagra-Caro et al., 2006; Geoghegan \& Pontikakis, 2008).

Tendo em vista o contexto apresentado e as lacunas teóricas na bibliografia atinentes ao tema, este estudo visa a ampliar a compreensão sobre a TTUE, no Brasil, investigando, especificamente, elementos intervenientes em processos que envolvem patentes acadêmicas. Para tanto analisa-se o caso de uma instituição de ensino superior (IES) privada, a Pontifícia Universidade Católica do Rio Grande do Sul (PUCRS).

Este artigo estrutura-se em sete seções. Dessa forma, na sequência a esta introdução ao tema, as seções dois e três apresentam uma revisão de literatura sobre a TT no contexto universitário e os intervenientes na TTUE, respectivamente; a seção quatro aborda os procedimentos metodológicos adotados na investigação; a cinco apresenta a instituição pesquisada; a seis, as análises e resultados do estudo; e, por último, são elencadas as considerações finais do trabalho. 


\section{TT no Contexto Universitário}

Compreende-se TTUE como um processo que pode envolver uma série de etapas, entre elas: (a) descoberta científica; (b) revelação da invenção pelos pesquisadores ao ETT da universidade (ou via comercialização e TT informais); (c) avaliação da invenção para patenteamento pelo ETT; (d) depósito de patente pelo ETT (se avaliação favorável), envolvendo os pesquisadores; (e) marketing/oferta da tecnologia por pesquisadores e ETT para empresas ou empreendedores; (f) negociação da licença; e (g) comercialização formal (ou informal) (Siegel, Waldman, Atwater, \& Link, 2004).

Após o Bayh-Dole Act, vários estudos passaram a considerar o licenciamento de patentes como sinônimo de TT, provocando um crescimento de ETTs em diversos países (Póvoa, 2008). Como mecanismos institucionais, os mesmos têm sido criados para atuar em instituições que enfocam, sobretudo, a comercialização de ativos intangíveis. Este é o caso da maioria dos países da Organization for the Economic Cooperation and Development (OCDE, 2003), que adotam a noção de ETT centrada na atuação em PI e todas as atividades decorrentes de sua identificação, proteção e exploração, abrangendo de projetos de $\mathrm{P} \& \mathrm{D}$ financiados por empresas a licenciamento de patentes.

O processo de TTUE pode ocorrer de várias maneiras, seja através da comunicação oral, da transferência física de um resultado de pesquisa tangível ou da complexidade de um programa de licenciamento da propriedade intelectual (Parker \& Zilberman, 1993). Estas e outras formas de TT, entretanto, não serão exploradas neste estudo, dado o seu enfoque específico em processos envolvendo patentes.

No Brasil, verifica-se o aumento da importância da gestão da PI e da TT formalizada no meio acadêmico, evidenciada pela existência dos Núcleos de Inovação Tecnológica (NIT), por políticas universitárias e desempenhos ascendentes (Guarnica \& Torkomian, 2009). Para a expansão da TTUE faz-se necessária, no entanto, uma sólida política nacional que respalde a disseminação da PI e promova a interlocução das universidades, governo e setor produtivo (Corrêa, 2007), dessa maneira, unindo esforços para superar dificuldades que este processo enfrenta.

Uma dessas dificuldades é a inexistência de uma legislação que uniformize a operacionalização dos ETTs. As políticas nacionais de incentivo à cooperação universidade-empresa (U-E) são recentes e há grande diversidade de procedimentos e de critérios para definir formas de licenciamento, para fixar preços ou royalties (Fujino \& Stal, 2007; Santos \& Solleiro, 2006).

Quanto ao setor produtivo, praticamente, não há investimentos em áreas estratégicas geradoras de alta tecnologia, tais como as de semicondutores, farmacêutica e química, passíveis de desenvolverem produtos que as incorporem (Corrêa, 2007). Tal fato constitui-se em uma barreira à TTUE, pois a capacidade de absorção empresarial é essencial para a incorporação de novos produtos, equipamentos, materiais e protótipos (Corrêa, 2007; Silva, 2007), sendo estes canais de TT mais fortemente correlacionados com patentes (Póvoa, 2008). Inexiste, tampouco, uma cultura empresarial para utilizar universidades como fontes tecnológicas (Santana \& Porto, 2009). Além disso, as tecnologias acadêmicas exigem muitos esforços e investimentos para transformarem-se em produto final, pois tendem a ser embrionárias (Póvoa \& Rapini, 2010).

No que tange às características da interação U-E, destacam-se: o predomínio de interações com grandes e médias empresas; a iniciativa de busca de pesquisadores pelas empresas, na maioria dos casos, via relações informais; poucos acordos de licenciamento (menos de $0,7 \%$ ) ou de transferência de know-how (8,5\%); e a relevância de empresas incubadas e do ambiente de inovação (Santos \& Solleiro, 2006).

Uma mudança recente foi o aumento do interesse dos pesquisadores do país por patentes acadêmicas, o que contribuiu para o seu rápido crescimento a partir de 2002. Este interesse foi influenciado por fatores, tais como: a criação dos ETTs nas universidades; o aumento de pesquisadores em doutorados/estágios no exterior; a difusão de casos de sucesso de gestão de patentes 
por universidades dos EUA; o crescimento da literatura sobre proteção e exploração de direitos de PI na academia; trocas de informações entre instituições via internet; e discussões sobre a nova Lei de Patentes (Póvoa, 2008).

\section{Intervenientes no Processo de TTUE}

A presente seção compreende uma síntese de resultados de pesquisas envolvendo intervenientes em processos de TTUE, tendo como enfoque a TT por meio de patentes, apresentando um paralelo entre dados de estudos empíricos internacionais e nacionais.

Observou-se a motivação primária de pesquisadores para a TTUE associada ao seu reconhecimento pela comunidade científica em alguns estudos internacionais. Embora as políticas de incentivo a patentes baseiem-se em royalties, Baldini, Grimaldi e Sobrero (2007) identificaram que prestígio, visibilidade e reputação, além de novo estímulo para pesquisas, estavam entre os seus principais motivadores, exemplificando este aspecto.

As motivações secundárias dos pesquisadores estiveram ligadas à obtenção de fundos de pesquisa adicionais para manter estudantes e equipamentos dos seus laboratórios, bem como a ganhos financeiros (Link, Siegel, \& Bozeman, 2007; Siegel et al., 2004). Um percentual de royalties maior para acadêmicos envolvidos em TTUE tende a estimulá-los; recompensas inadequadas são, por outro lado, uma barreira à eficácia deste processo (Baldini, 2010; Caldera \& Debande, 2010).

No Brasil, Santana e Porto (2009) revelaram, de modo semelhante, que a falta de recompensa para o professor é um desmotivador para a TTUE. Já a possibilidade de obter recursos financeiros, maior interação com o mercado e melhor utilização de equipamentos (Segatto-Mendes \& Mendes, 2006) foram aspectos apontados como motivadores.

Entre os principais determinantes para o desempenho da TTUE, estão as políticas institucionais e os procedimentos para o gerenciamento da TT das universidades (Caldera \& Debande, 2010). Instituições com regras internas para patenteamento demonstram aos pesquisadores seu empenho em promover uma mudança cultural, em desenvolver um ambiente empreendedor e legitimar esta atividade (Baldini, Grimaldi, \& Sobrero, 2007). Segundo Caldera e Debande (2010), regras que tratam dos conflitos de interesses entre as responsabilidades de ensino e as atividades externas dos acadêmicos afetam positiva e significativamente o desempenho universitário em contratos de P\&D e licenciamentos.

Dada a carência de regras nesse sentido, no Brasil, há casos de demora e ausência de envio de relatórios descritivos de patentes devido à sobrecarga de atividades dos docentes (Santana \& Porto, 2009). Ademais, as políticas institucionais de avaliação e de fomento à pesquisa do governo valorizam, quase que exclusivamente, publicações científicas, e não a geração de patentes por parte dos pesquisadores (Amadei \& Torkomian, 2009; Corrêa, 2007).

Observa-se, mesmo no contexto internacional, ainda, a existência de barreiras culturais e informacionais no processo de TTUE, tanto por parte de acadêmicos como de administradores das universidades e de empresas/empreendedores (Siegel et al., 2004). No Brasil, pesquisadores relataram dificuldades quanto à falta de informação sobre como proceder para patentear algo e a necessidade de adaptar a linguagem da descoberta para os moldes de redação do pedido de entrada no INPI (Corrêa, 2007). Além disso parcerias com empresas ainda não são valorizadas pela cultura acadêmica do país, dificultando, dessa forma, a adoção de políticas de licenciamento e comercialização de tecnologias (Fujino \& Stal, 2007).

Há também dificuldade para a obtenção de financiamentos no Brasil (Segatto-Mendes \& Mendes, 2006) e a aplicação de recursos financeiros dos órgãos de fomento é voltada, prioritariamente, para a pesquisa teórica, pois a pesquisa aplicada é mais onerosa e requer laboratórios 
(Corrêa, 2007). Ademais, existe carência de verbas para construir protótipos que tangibilizem resultados de pesquisa e facilitem sua comercialização (Santana \& Porto, 2009).

As características institucionais das universidades, igualmente, intervêm na TTUE (Caldera \& Debande, 2010). A burocracia e a inflexibilidade universitárias são barreiras neste processo, gerando, assim, insatisfações que desviam pesquisadores e membros de empresas dos ETTs, levandoos a estabelecer relações informais e de consultoria (Siegel et al., 2004). Tal como no exterior, a burocracia administrativa, a morosidade da área jurídico-administrativa para efetivar contratos e a dificuldade em fixar percentuais de royalties para repasse à universidade, aliadas à rigidez das regulamentações de órgãos públicos do país, acabam, por vezes, excluindo as universidades do processo formal de TT e do compartilhamento de seus resultados (Santana \& Porto, 2009).

Os intermediários na TT, entre eles, os ETTs, são igualmente importantes para a TTUE (Caldera \& Debande, 2010). O sucesso deste processo reside, em grande parte, em atributos dos ETTs, incluindo a sua autonomia institucional e a experiência da sua equipe (Geoghegan \& Pontikakis, 2008). Conforme Caldera e Debande (2010), ETTs maiores e mais experientes podem obter mais contratos de pesquisa, conquanto isso não afete sua receita de licenciamentos.

Segatto-Mendes e Mendes (2006) evidenciaram a importância do apoio dos ETTs para o patenteamento e comercialização de tecnologias no Brasil, podendo, dessa forma, facilitar a solução de questões burocráticas. Demoras no processo de patenteamento, contudo, foram verificadas quando havia um corpo exíguo de colaboradores nesses escritórios (Santana \& Porto, 2009).

Com respeito às habilidades de marketing e negociação das equipes dos ETTs, pesquisadores e empresas mostram-se insatisfeitos em âmbito mundial (Siegel et al., 2004). De acordo com Lester (2005), as universidades necessitam conhecer os rumos que a indústria local está tomando, seus processos de inovação, suas forças e fraquezas, buscando alinhar suas contribuições ao que o mercado requer. Segundo Golish, Besterfield-Sacre e Schuman (2008), definir o mercado e seu potencial de crescimento, identificar mudanças nas necessidades de mercado e custos podem auxiliar a gerar mais licenciamentos e patentes. A comercialização de PI pode ser difícil em razão de que novas tecnologias possuem valor para poucas organizações e somente quando aplicadas. As tecnologias acadêmicas, no entanto, raramente podem ser aplicadas diretamente e é penoso para um comprador potencial avaliar seu valor antes de investigá-las, testá-las e adotá-las (Elfenbein, 2007).

Póvoa (2008) salienta que conhecimentos profundos do campo técnico e de suas aplicações, bem como habilidades negociais para realizar contratos complexos com indústrias, são necessários à eficácia da TTUE, embora tais competências sejam raras entre administradores de Universidades. Há também falta de autonomia e infraestrutura adequada às operações dos ETTs de IES públicas no Brasil, em geral, unidades internas, com funcionários que não recebem remuneração satisfatória ou compartilham royalties que os estimulem a viabilizar a TT. Além disso, há uma distância entre os seus objetivos de marketing e suas ações, pouco atentas às demandas de mercado. Os sites dos ETTs universitários, normalmente, são de difícil acesso e sem links para informações sobre patentes disponíveis (Fujino \& Stal, 2007).

Castro, Jannuzzi e Mattos (2007) assinalam alguns aspectos positivos do funcionamento ETT da UNICAMP, considerado modelo de atuação no Brasil: contratação de escritórios especializados em marcas e patentes; banco de patentes disponível em sua página da internet; experiência acumulada de seus integrantes; práticas de negociação e gestão empresarial que consideram autossustentabilidade, cumprimento de metas e de resultados; atuação pró-ativa, em parceria com os pesquisadores; mescla de experiência acadêmica e comercial pela equipe.

Os ETTs dependem da informação dos pesquisadores sobre suas descobertas comercializáveis, o que não ocorre, às vezes, por falta de percepção deste potencial. Alguns pesquisadores, no entanto, simplesmente, não estão dispostos a se envolver em licenciamento por falta de interesse, por considerarem difícil trabalhar com o ETT, ou por não quererem compartilhar rendimentos com a 
universidade (Thursby, Fuller, \& Thursby, 2009), constituindo-se, dessa maneira, em barreiras ao processo de transferência de tecnologia.

Parques tecnológicos nas universidades são também intermediários na TTUE. No papel de incubadoras, reduzem custos relativos à conversão de achados científicos em produtos ou processos comercializáveis que favorecem a sua comercialização. A aproximação geográfica viabiliza, ainda, a transferência de conhecimento tácito entre pesquisadores e profissionais das empresas, apresentando impacto positivo no sucesso comercial do invento (Agrawal, Kapur, \& McHale, 2008). Ademais, a concentração local de empresas high-tech possibilita a valorização da pesquisa acadêmica (Caldera \& Debande, 2010).

O papel dos atores envolvidos na TTUE, sobretudo o dos inventores, é crítico nesse processo. Estudos internacionais destacam o papel dos pesquisadores desde a comunicação do invento à sua comercialização (Agrawal, 2006; Thursby et al., 2009). De acordo com Agrawal (2006), novas tecnologias podem ser complexas, combinando disciplinas aprendidas em distintas áreas de conhecimento, a partir das necessidades dos pesquisadores, competências incorporadas à invenção que os licenciados podem não ter.

Os inventores desenvolvem uma intuição que facilita prever como o invento irá se comportar, advinda do aprendizado de conhecimentos latentes adquiridos, por exemplo, a partir de erros, que não constam em nenhum registro. Por este e outros fatores, o envolvimento do inventor no processo de desenvolvimento de uma inovação aumenta a probabilidade de seu sucesso comercial (Agrawal, 2006).

No contexto nacional, Guarnica e Torkomian (2009) salientaram a importância do inventor no marketing da tecnologia, além da sua colaboração para transferir conhecimento tácito para as empresas. Conforme Silva (2007), a co-produção de tecnologia é positiva, ao invés da simples encomenda de um produto, pois o processo de elaboração e aperfeiçoamento de tecnologias é dinâmico e exige redirecionamentos.

Bercovitz e Feldman (2008) evidenciaram a importância da aprendizagem social no ambiente de trabalho destacando que: indivíduos com vivências em instituições ativas em TT tendem a ser mais participativos nesse processo; quando o chefe da unidade se envolve em TT, outros membros tendem a se envolver também; acadêmicos, em geral, seguem o envolvimento em TT de pares com os quais se identificam; indivíduos confrontados por normas dissonantes entre experiências prévias e atuais com relação à TT tendem a seguir as normas do trabalho atual. Casos de sucesso são também importantes para incentivar o patenteamento e licenciamento, mas a atenção exclusiva a esses inventos pode desestimular outros pesquisadores nessas atividades (Baldini, 2006).

No Brasil, experiências anteriores positivas de TTUE incentivaram novas tratativas (Santana \& Porto, 2009); a pressão do tempo e desconfianças com relação ao tempo necessário para a universidade atingir suas metas e quanto à segurança e confidencialidade das informações, por sua vez, foram barreiras a esse processo (Cruz \& Segatto, 2009).

Relacionamentos pessoais podem ser mais relevantes para a TTUE do que contratos, o que confere importância ao desenvolvimento de networks entre pesquisadores acadêmicos e de empresas, diretores de IES e de ETTs (Siegel et al., 2004). A confiança entre membros de universidades e de empresas é essencial e requer investimentos em relacionamentos de longo prazo, entendimento dos diferentes sistemas e objetivos, além de contatos face a face, sobrepondo relacionamentos pessoais e profissionais (Bruneel, D’Este, \& Salter, 2010).

Alguns destes aspectos, relativos a relacionamentos e comunicação U-E, assemelham-se em estudos brasileiros, entre eles: a necessidade de desenvolver confiança (Santana \& Porto, 2009) e linguagem comum; a percepção da natureza distinta do trabalho dos parceiros (Silva, 2007); e a solução de conflitos pela intensificação das relações, em encontros e diálogos informais, favorecidos pela vivência de membros da empresa em cursos realizados no ambiente acadêmico (Segatto-Mendes \& Mendes, 2006; Silva, 2007). 
Hoye e Pries (2009) analisaram um grupo de inventores reincidentes na comercialização de tecnologias universitárias (12\% deles contabilizavam $80 \%$ das inovações comercializadas). Suas características eram: habilidade comercial (gerar, identificar inventos comercializáveis e reunir esforços para comercialização); atitude favorável à comercialização; interação com a indústria; aprendizagem com experiências comerciais prévias; alta produção acadêmica.

Diversos estudos internacionais observaram que os pesquisadores que mais patenteiam inventos são também os que mais produzem publicações (Hoye \& Pries, 2009). Segundo Elfenbein (2007), o status acadêmico de um inventor atrai licenciadores potenciais. Registros de inventos acadêmicos, normalmente, impactam positivamente a obtenção de verbas do governo e da indústria (Thursby \& Thursby, 2010), contribuindo para que o envolvimento em TTUE possa aumentar a quantidade e a qualidade da pesquisa básica (Siegel et al., 2004).

Apesar da importância da alta qualidade acadêmica para a TTUE (Silva, 2007), a capacitação científica, por si só, não pode ser associada diretamente à geração de patentes ou à TT no Brasil. Há áreas com maior número de grupos de pesquisas e menor interação com empresas no país, bem como IES com muitos pesquisadores e publicações, mas pouco expressivas em registros de patentes (Amadei \& Torkomian, 2009; Rapini \& Righi, 2006).

\section{Procedimentos Metodológicos}

O presente estudo objetivou compreender motivadores, obstáculos e facilitadores em processos de TTUE envolvendo patentes. Para tanto, adotou uma abordagem qualitativa de pesquisa, cuja orientação favorece a compreensão de processos (Denzin \& Lincoln, 2005). A pesquisa realizada foi de caráter exploratório, que permite desenvolver, esclarecer e modificar conceitos e ideias visando a formular problemas e hipóteses para investigar posteriormente (Gil, 1999). Este estudo, do tipo descrito, tem como característica "resumir dados qualitativos através de abstrações na forma de categorias a fim de considerar possíveis relações para a descrição mais exata em pesquisas ulteriores" (Tripodi, Fellin, \& Meyer, 1981, p. 65).

O método utilizado, em virtude do caráter exploratório da pesquisa, foi o estudo de caso (Tripodi et al., 1981). Para atender aos objetivos da pesquisa, além da revisão de literatura e da consulta a documentos da Pontifícia Universidade Católica do Rio Grande do Sul, foram realizadas entrevistas com sete pesquisadores e com a gestora do ETT desta universidade, considerada, no Brasil, uma referência em pesquisa e no processo de TTUE.

Os pesquisadores foram selecionados por sua relevância e representatividade no meio acadêmico em suas áreas de pesquisa, bem como pelo número de patentes requeridas ao ETT da PUCRS. A identificação dos mesmos foi realizada a partir deste escritório, que faz a gestão da propriedade intelectual gerada na universidade.

A coleta de dados foi realizada mediante entrevistas em profundidade a partir da formulação de perguntas abertas. Para a elaboração do roteiro de entrevista foram identificados fatores intervenientes no processo de TTUE, envolvendo patentes na revisão de literatura internacional e nacional. Este roteiro procurou caracterizar o histórico do projeto que resultou em patente, bem como os intervenientes no processo, entre eles, as motivações que levaram ao início da pesquisa, os obstáculos e os facilitadores nesse processo. O conteúdo do roteiro de entrevista foi validado por especialistas. Na Tabela 1, descrevem-se as áreas de atuação e o número de patentes requeridas pelos pesquisadores entrevistados. 
Tabela 1

Áreas de Atuação e Número de Patentes Requeridas pelos Pesquisadores Entrevistados

\begin{tabular}{llc}
\hline \multicolumn{1}{c}{ Entrevistado } & \multicolumn{1}{c}{ Áreas de Atuação } & Patentes \\
\hline Pesquisador 1 & Biologia Celular e Molecular & 2 \\
Pesquisador 2 & Biologia Celular e Molecular & 7 \\
Pesquisador 3 & Farmácia Aeroespacial & 3 \\
Pesquisador 4 & Imunologia Celular e Molecular & 3 \\
Pesquisador 5 & Bioquímica & 2 \\
Pesquisador 6 & Microgravidade & 6 \\
Pesquisador 7 & Biologia Celular e Molecular, Medicina e Ciências da Saúde & 4 \\
\hline
\end{tabular}

As entrevistas foram gravadas com a autorização dos entrevistados e posteriormente transcritas. A análise dos dados foi realizada por meio da análise de conteúdo, técnica que consiste em descrever, de modo objetivo, sistemático e quantitativo, o conteúdo efetivo de uma comunicação (Bardin, 1977; Malhotra, 2001), ou seja, procura uma maneira sistematizada para trabalhar dados desestruturados, diminuindo, com isso, a dependência da subjetividade do analista (Freitas, Cunha, \& Moscarola, 1996). Para melhor analisar as repostas, os dados foram agrupados em grandes categorias, definidas, inicialmente, de acordo com os fatores intervenientes na TTUE, quais sejam: motivadores; políticas institucionais, características e procedimentos; intermediários no processo; e papel crítico dos inventores. Os resultados encontrados são descritos e analisados na seção seis.

\section{Caso PUCRS: a Visão Institucional}

A PUCRS é reconhecida no país como uma universidade empreendedora e inovadora, tendo inclusive recebido o Prêmio FINEP de Inovação na Categoria Instituição de Ciência e Tecnologia, obtendo o primeiro lugar na Região Sul, em 2008, e Menção Honrosa Nacional, no mesmo ano. Para a PUCRS, a inovação é um processo estratégico do qual fazem parte busca, descoberta, experimentação, desenvolvimento e adoção de novos produtos, processos ou técnicas organizacionais capazes de agregar valor às organizações (Audy \& Ferreira, 2006).

Em 2006, foi estruturada na universidade a Rede INOVAPUC, que tem como objetivo promover o processo de inovação e empreendedorismo na Universidade, articulando, para tal, todos os atores envolvidos no ensino, pesquisa e extensão. A universidade visa a fomentar um esforço multidisciplinar para buscar soluções e respostas às demandas de desenvolvimento econômico, social, ambiental e cultural da sociedade (Audy \& Ferreira, 2006).

Dessa forma, a relação entre universidade e sociedade pode ocorrer em dois sentidos: tanto problemas identificados na sociedade podem dar origem ao desenvolvimento de pesquisas quanto resultados e conhecimento já disponíveis na universidade podem ser aplicados na solução de problemas existentes. Neste contexto, a geração de patentes é um mecanismo de transferência de tecnologia, entre outros, a partir do qual o conhecimento gerado na universidade pode ser apropriado pela sociedade (Póvoa, 2008).

A partir da necessidade de proteger o patrimônio intelectual da universidade e promover a transferência dos resultados de pesquisa ao setor produtivo, foi criado, em 2005, o ETT da PUCRS. Entre os objetivos institucionais deste escritório, divulgados em seu site, estão estabelecer e promover a política institucional de PI e TT, além de implementar e divulgar os procedimentos necessários à proteção da PI dos resultados de pesquisas realizadas nas diferentes unidades da Instituição, bem como 
os relacionados à TT através da comercialização de ativos, protegidos ou não, de propriedade da PUCRS.

Assim, de acordo com a resolução interna de $n^{0}$ 001/2007, que estabelece as diretrizes da Política Institucional de PI e TT da PUCRS, todos os pesquisadores da universidade devem comunicar ao ETT os achados de pesquisa que têm potencial de proteção da propriedade intelectual e transferência à sociedade. O ETT, por seu turno, fornece o suporte para todo o processo de proteção e negociação da transferência de tecnologia quando for o caso. Esta estrutura conta com profissionais das áreas técnicas e especializados na área.

No que se refere às atividades de registro da PI, o ETT tem como atribuição avaliar a invenção, desde o ponto de vista de sua viabilidade técnica e econômica, visando ao adequado registro nos órgãos competentes do país e do exterior. O encaminhamento dos pedidos se dá através de formulários específicos disponíveis no site do escritório. Após a identificação da demanda, são realizados procedimentos internos, tais como buscas em base de patentes e análise da viabilidade técnica e econômica do invento, no caso de patentes. Além dos trâmites administrativos junto aos órgãos responsáveis pelo registro da PI, após o depósito dos pedidos, o ETT monitora os processos com vistas ao adequado cumprimento dos prazos de pagamento e demais obrigações da PUCRS, enquanto titular dos ativos intangíveis.

$\mathrm{Na}$ área da Pesquisa e Desenvolvimento (P\&D) realizada em parceria com empresas privadas, o papel do ETT é participar das negociações dos projetos, especificamente, no que se refere à PI e ao sigilo, cabendo-lhe assegurar que os direitos da PUCRS sejam preservados, de acordo com o que estabelecem as políticas institucionais que regem a matéria.

\section{Principais Intervenientes no Processo de TTUE no Caso PUCRS}

Esta seção apresenta a análise dos principais intervenientes no processo de TTUE da PUCRS. Destaca-se, inicialmente, que o produto tecnológico não foi premeditado para a maioria dos seus pesquisadores produtores de patentes, mas consequência da própria pesquisa, tal como verificado em outros estudos realizados no Brasil (Corrêa, 2007; Póvoa, 2008). Assim, entre os principais intervenientes no processo de TTUE, estiveram as próprias motivações dos pesquisadores para a geração de pesquisas, como explicitado a seguir.

A busca por soluções para problemas foi um dos principais motivadores para a geração de pesquisas que resultaram em pedidos de patentes, seja para atender necessidades específicas do pesquisador, do laboratório ou demandas empresariais e sociais. Os entrevistados compartilharam a visão de que "a necessidade é a mãe do processo de invenção", como expressou o pesquisador 1, que relatou, tal como outros pesquisadores da PUCRS, sentir-se estimulado por desafios científicos.

O reconhecimento social pela geração de benefícios públicos e entre pares foi outro motivador para a geração de patentes entre os pesquisadores da PUCRS, assim como entre pesquisadores da Itália (Baldini et al., 2007). Conforme a gestora do ETT da PUCRS:

"Se tu conseguires associar prestígio acadêmico com ganho econômico e além de tudo o reconhecimento da sociedade pela tua contribuição em relação a esse produto, ou seja, atuando diretamente na melhoria das condições das pessoas e tal ... sem dúvidas isso vai causar uma repercussão, um impacto e um desejo maior dos pesquisadores de atuar nessa área".

Conforme o relato descrito, os ganhos econômicos associam-se a outros fatores que motivam a TTUE. A possibilidade de obter verbas adicionais para equipamentos, reagentes e alunos, entre outros recursos para realizar pesquisas de laboratório, foi muito valorizada pelos pesquisadores da PUCRS, tal como em outros casos investigados (Link et al., 2007; Santana \& Porto, 2009; Segatto-Mendes \& Mendes, 2006; Siegel et al., 2004). 
O patenteamento de resultados de pesquisa e a visão das possibilidades de ganhos incentivaram também certa competição entre os pesquisadores, gerando novas tentativas de patenteamento. A motivação para realizar pesquisas que resultaram em patentes foi ainda fruto da necessidade de produzir artigos científicos, um dos principais quesitos avaliados por órgãos de fomento à pesquisa do governo para aprovação de verbas com este fim.

Um interveniente significativo no processo de TTUE da PUCRS é a sua política institucional, que tem o empreendedorismo e a inovação entre seus pilares de gestão, tal como expressa seu estatuto (aprovado pela Portaria ${ }^{\circ}$ 597, de 13 de setembro de 2006, da Secretaria de Educação Superior do Ministério da Educação). Esta política gera um ambiente acadêmico favorável à TTUE, valorizado pelos entrevistados e também destacado em estudo realizado por Santos e Solleiro (2006). A importância das políticas das IES é, ainda, assinalada no contexto de países europeus que estão, como o Brasil, buscando melhorar a sua performance nesse processo (Baldini et al., 2007; Caldera \& Debande, 2010).

A integração da proteção à PI ao estatuto e ao regimento da PUCRS demonstra a preocupação da instituição com esse aspecto que, segundo Baldini et al. (2007), contribui para promover uma mudança cultural e para legitimar esta atividade. Diferente do que ocorre na maioria das IES públicas nacionais (Stal \& Fujino, 2005), as políticas institucionais acadêmicas da PUCRS valorizam claramente a pesquisa, a PI e a TTUE, havendo incentivos e apoio efetivo para que os pesquisadores gerem patentes, o que gratifica e motiva a geração de novas pesquisas, como sinaliza a fala da pesquisadora 4:

"A PUC começou com a política de incentivo às patentes ... é muito gratificante porque eles incentivam, tu vai buscar o incentivo e assim, realmente, eles não faltaram. ... O que facilitou nesses estudos, eu acho que foi uma mentalidade geral da PUC de apoiar os pesquisadores ... o que eu ouvi foi: 'vai, a gente te dá apoio"”.

A dificuldade de conciliar o tempo entre pesquisa, processo de patenteamento e docência, entretanto, foi um entrave para os pesquisados, evidenciado também por Santana e Porto (2009). Caldera e Debande (2010) observaram que regras para lidar com tais conflitos de interesses favorecem o desempenho universitário em contratos de P\&D e licenciamentos.

O tempo e o custo elevado para registro e manutenção de patentes foram igualmente entraves ao processo de TTUE, além da necessidade de adaptação da linguagem da descoberta para os moldes de redação do pedido para dar entrada no INPI, aspectos corroborados por Corrêa (2007) em estudo realizado no contexto nacional. Segundo Silva (2007), o processo de TTUE precisa ser controlado e institucionalizado pelas IES para que alguns docentes possam se dedicar mais a ele por um tempo e retomar, posteriormente, às suas atividades habituais.

A importância de cumprir as metas estipuladas pela PUCRS, com foco no desenvolvimento do empreendedorismo e da inovação foi salientada pelo pesquisador 2. Para atendê-las, o mesmo fez cursos disponibilizados pela própria universidade, na faculdade de Administração, adequando-se para atender esses propósitos. É perceptível a mudança cultural que vem ocorrendo na universidade, promovendo a geração de patentes e a TTUE, estimulada pela alta direção, como demonstra o relato da pesquisadora 3 :

"Quando eu saí da Puc, tinha um sistema antigo, quando eu retornei era um outro sistema, então passei pela adaptação que todo mundo passa e agora estou conseguindo interagir mais e ver essa história do mercado ... existe contratos com empresas para nós tentarmos dentro dessa possibilidade".

Alguns entrevistados, no entanto, expressaram barreiras à TTUE relacionadas à cultura acadêmica, em geral, que ainda concebe a ciência aplicada como de "segunda categoria"; valoriza publicações, mas não patentes; e possui pouco conhecimento e/ou informações errôneas sobre o processo de patenteamento, aspectos observados também por Corrêa (2007). 
A fala da pesquisadora 4 sintetiza alguns obstáculos à TT relacionados à falta de comunicação e informação, no que tange à interação universidade-empresa, bem como ao perfil de atuação das empresas, geralmente avessas ao risco:

"A gente não sabe o que a indústria precisa e a indústria não sabe o que a gente pode fazer, no Brasil. Segundo, a indústria não tem investido no Brasil (não tem investido em patente, ela não sabe o potencial da universidade, que tem gente muito boa) então eu entendo essa coisa de risco, não existe uma tradição no Brasil, então, assim, eles não estão acostumados, eles querem uma coisa certa".

Por sua vez, há uma preocupação que a excessiva valorização comercial da ciência aplicada possa limitar a geração de novas ideias, como exprime a pesquisadora 6.

"A aplicação da ciência, eu acho que isso é uma maneira muito pobre de ver a ciência, a ciência te dar o lucro. ... eu acho é que isso não pode ser um fator limitante, que eu só posso pensar naquilo que dá dinheiro. Se eu fizer isso eu estou aniquilando com milhões de ideias, que podem até, no início, não dar dinheiro, aparentemente, e depois dar muito dinheiro".

Líderes de grupos de pesquisas do Brasil, no entanto, relataram ganhos acadêmicos em quase todas as suas relações de TTUE, o que indica que a academia não está se desviando de seu foco principal em favor de ganhos econômicos (Póvoa, 2008). Thursby e Thursby (2010) observaram ainda um crescimento no licenciamento de inventos patenteados, mais associado ao aumento na inclinação dos professores para registrar patentes e das empresas para terceirizar atividades de $P \& D$, através de licenciamentos, do que à tendência de mudança das pesquisas, de básica à aplicada.

Conforme a gestora do ETT entrevistada, é preciso "manter a base de pesquisa básica forte porque é ela que vai dar sustentação para desenvolver o resto". Segundo ela,

"a universidade tem lugar para todas as formas de atuação, não precisa todo mundo só fazer pesquisa básica nem todo o mundo só fazer pesquisa aplicada ... se a gente souber preservar esses vários nichos dentro da universidade, essas várias funções dentro da universidade ao mesmo tempo, eu não vejo risco nenhum".

O acesso à informação, sobretudo em bases acadêmicas internacionais, foi a principal fonte inicial de pesquisa. Este aspecto foi muito valorizado pelos pesquisadores, assim como a estrutura dos laboratórios disponibilizados pela PUCRS e a preocupação da universidade em atrair professores e alunos qualificados. A consulta às bases de patentes, em geral, deu-se apenas no momento anterior ao pedido de depósito de patente.

A importância do conhecimento constituído por meio de grupos de pesquisa consolidados - em consolidação, no caso da PUCRS - foi salientada. A integração de visões de distintas áreas, em grupos de pesquisa multidisciplinares, segundo os entrevistados, favoreceu a criação de novos conhecimentos que resultaram em patentes. Tal integração é descrita, por Nonaka e Takeuchi (1997), como uma das condições para a criação do conhecimento nas organizações.

O pesquisador 2 observou que gerar recursos para a universidade é uma tendência mundial, mas frisou as diferenças de atitude entre professores de IES públicas e privadas. Nestas últimas, como é o caso da PUCRS, há maior exigência de comprometimento dos pesquisadores com as políticas e os objetivos da universidade.

A estrutura de suporte administrativo e legal da PUCRS, burocrática e centralizadora, é um aspecto que dificulta o fluxo dos processos envolvidos na TTUE, mencionado por vários entrevistados. Segundo Castro et al. (2007), um ETT independente do processo decisório e com visão de autossustentabilidade financeira favorece esse processo.

Com respeito a intermediários na TTUE, estruturas específicas, como o ETT, que apoia o processo de patenteamento e a AGT (Agência de Gestão Tecnológica), que auxilia a criação e gestão de contratos, foram muito valorizados pelos pesquisadores, bem como a contratação de consultores externos. Mas ainda existe uma lacuna no que tange à comercialização, conforme evidencia o 
depoimento da pesquisadora 6: “o ETT faz a parte burocrática, nós fazemos o desenvolvimento. E aí a comercialização tem que ter, tem que criar o esforço".

Os entrevistados relataram dificuldades em valorar resultados de pesquisa, em negociar com empresas e em identificar e se aproximar de parceiros comerciais interessados em licenças/patentes. Por lidar com um produto intangível, alguns obstáculos são inerentes a este mercado, tornando tais dificuldades globais (Elfenbein, 2007; Siegel et al., 2004).

A localização do parque tecnológico da PUCRS, o Tecnopuc, no campus universitário, possibilita uma proximidade física entre as indústrias e os pesquisadores, um fator importante para a efetivação de inovações, segundo os entrevistados, em especial, se o desenvolvimento da tecnologia demandar conhecimento tácito dos pesquisadores (Agrawal et al., 2008; Lipinski, Minutolo, \& Crothers, 2008).

Outro fator crítico é o papel dos inventores na TTUE. Os entrevistados salientaram características dos pesquisadores que favoreceram a geração de pesquisas que resultaram em patentes. Entre elas, a criatividade - característica culturalmente associada aos brasileiros - para buscar soluções de problemas de pesquisa, a persistência e a curiosidade.

Alguns entrevistados frisaram que o conhecimento é pré-requisito para originar pesquisas inovadoras, geradoras de patentes, dando ênfase à formação do pesquisador, processo que envolve o intercâmbio entre práticas formais e informais de aprendizagem:

“A ideia surge ... depois que tu vê milhões de museus, lê vários livros, viaja muito, conhece lugares. Eu acho que depois de tanta exposição ... em congressos, mundo afora ... esse conhecimento ele te aflora numa ideia. É uma comunhão de aspectos, é tu saber que aquele aspecto específico não foi ainda investigado, saber como buscar o que já foi feito até aquele momento, ver a necessidade" (Pesquisadora $6)$.

Como a geração de patentes e a TTUE são bastante recentes na PUCRS, a maioria dos pesquisadores entrevistados aprendeu sobre esses processos com modelos de atuação de universidades e de pesquisadores em vivências no exterior. O mesmo ocorreu com outros pesquisadores brasileiros (Mariz, 2009), que relataram, a partir dessas vivências, terem mudado seus modelos e valores com relação à pesquisa aplicada.

As experiências dos pesquisadores e funcionários pioneiros no processo de TTUE na PUCRS propiciaram novos aprendizados e abriram novos caminhos passíveis de serem reproduzidos em novas situações, propiciando, desse modo, gratificações que estimularam mais pesquisadores a realizarem tentativas nesse sentido. Tais aspectos revelam a contribuição da aprendizagem social (Bercovitz \& Feldman, 2008) para o sucesso da TTUE na PUCRS. Assim, a oportunidade de obter verbas com empresas, através da comercialização de patentes, começou a repercutir entre os pesquisadores da PUCRS a partir do licenciamento da primeira patente. O pesquisador 2 expressou essa importância.

"É muito importante licenciar a primeira patente. Pra mostrar que é possível. Tem um caso ... que além de gerar patente, parte do desenvolvimento vai ser feito aqui na Puc ... vai vir um dinheiro pro meu laboratório, com aluno, vai ter bolsa para mim”.

A participação em eventos internacionais e a aproximação com empresas também possibilitaram novas visões quanto aos benefícios da TTUE entre os entrevistados da PUCRS, estimulando-a. As experiências de interação universidade-empresa auxiliaram o processo de comunicação e a compreensão das distintas linguagens e objetivos, estreitando relações e aumentando a confiança mútua, aspectos identificados como relevantes à TTUE também em outros estudos nacionais (Cruz \& Segatto, 2009; Guarnica \& Torkomian, 2009).

A importância dos relacionamentos, destacada pelos entrevistados, é igualmente evidenciada em estudos internacionais (Bruneel et al., 2010; Siegel et al., 2004). Bons relacionamentos entre professores, alunos e técnicos auxiliaram o processo cooperativo na universidade para a geração de pesquisa e de patentes. Relações dos acadêmicos com integrantes do governo facilitaram a obtenção 
de recursos e a formação de parcerias para o fornecimento de produtos ou serviços. O vínculo de pesquisadores com membros de empresas (muitos ex-alunos) favoreceram a identificação de oportunidades e/ou necessidades entre as partes, facilitando a TTUE sob suas diversas formas. Por seus relacionamentos e pela familiaridade com o trabalho em sua área, os pesquisadores representam as melhores fontes para identificar potenciais parceiros comerciais (Lipinski et al., 2008).

O papel do governo na TTUE foi bastante destacado pelos entrevistados da PUCRS e corroborado por diversos pesquisadores brasileiros (Closs \& Ferreira, 2010), embora este aspecto não tenha sido salientado em estudos internacionais. As maiores fontes de recursos financeiros, segundo os pesquisados da PUCRS, são os órgãos de fomentos do governo (CAPES, CNPq, FINEP, BNDES). Estes recursos são considerados vitais ao processo de TTUE para adquirir insumos para pesquisa, construir protótipos e obter registros de patentes, bem como recursos físicos e humanos.

Esta importância do papel do governo na TTUE, no país, decorre da falta de articulação histórica entre as políticas governamentais industriais e de Ciência e Tecnologia (C\&T) no Brasil. Há uma concentração de investimentos públicos na área de ciência no contexto nacional e pouco investimento do setor privado em desenvolvimento tecnológico (Santos \& Solleiro, 2006). Com isso, os avanços significativos em produção científica alcançados pelo Brasil não têm se traduzido em seu desenvolvimento tecnológico.

No caso da PUCRS, além disso, a valorização da pesquisa científica é recente, sendo pouco comum este investimento em pesquisa, em outras universidades privadas no Brasil. Devido a isso, segundo a pesquisadora 5, ainda, existe um preconceito, por parte do governo, com relação à produção de pesquisas na PUCRS e uma destinação de verbas inferiores às universidades privadas, em comparação com as públicas, de um modo geral.

No entanto, segundo a gestora do ETT pesquisada, as políticas de apoio governamentais à TTUE, concebidas como vetores de desenvolvimento do país, vêm mudando, reduzindo esse e outros preconceitos. A lei de inovação, por exemplo, sinaliza que as universidades podem e devem interagir com empresas. Conforme a gestora do ETT, muito do comércio internacional baseia-se em PI e o INPI deu conta que grande parte da pesquisa nacional estava na academia, onde se gerariam patentes, o que provocou uma alteração em seus valores.

A visão de oportunidades para obtenção de recursos com a comercialização de patentes para empresas na PUCRS ainda é recente, embora venha sendo ampliada a partir do licenciamento das primeiras patentes. Segundo o pesquisador 1, no entanto, ainda "não existe uma cultura na empresa nacional de fazer essa conexão, de obter respostas a partir da academia". As interações, geralmente, deram-se com indústrias que atuam em mercados globais, sendo a primeira licença da PUCRS concedida para uma grande indústria farmacêutica nacional.

A síntese dos principais intervenientes no processo de TTUE envolvendo patentes em estudos internacionais, nacionais e no caso PUCRS são apresentados na Tabela 2. 
Tabela 2

Intervenientes no Processo de TTUE: Síntese de Resultados de Pesquisas Internacionais, Nacionais e Caso PUCRS

\begin{tabular}{llcl}
\hline Intervenientes & Estudos internacionais & Estudos Brasil & Estudo caso PUCRS \\
\hline Motivadores & . Reconhecimento pela & . Fundos para pesquisa; & . Busca de solução para \\
para os & comunidade científica; & interação com o mercado; & problemas; desafios \\
pesquisadores & fundos para pesquisa; & melhor uso de & cquíficos; reconhecimento \\
& ganhos econômicos. & recompensas. & social e entre pares; ganhos \\
& & & econômicos; fundos para \\
pesquisa; competição entre \\
pesquisadores; produção de
\end{tabular}

\section{Políticas, características institucionais $\mathbf{e}$ procedimentos para gerenciamento da TTUE}

\begin{abstract}
Ausência de regras p/patenteamento; barreiras culturais e informacionais; burocracia administrativa; morosidade jurídicoadministrativa; rigidez; regulamentação - processos informais de TT.
\end{abstract}

\section{Intermediários no processo de TTUE}

. ETT: autonomia e experiência da equipe, conhecimentos técnicos; marketing, negociação e conhecimento de mercado.

. Parques tecnológicos: positivo para pesquisas e comercialização de resultados.

\section{Papel crítico dos inventores no processo de TTUE}

\author{
Habilidade comercial; \\ atitude favorável à TT; \\ interação U-E; vivências, \\ atuação do chefe/pares, \\ reincidência na \\ comercialização; alta \\ produção acadêmica; \\ relacionamentos \\ pessoais/network, confiança.
}

. ETT: dificuldades com comercialização e questões burocráticas; falta de autonomia, infra-estrutura e remuneração inadequadas; distância entre objetivos de marketing e ações de mercado; desinteresse dos pesquisadores;

acessibilidade dos sites na internet, terceiros especializados, experiência da equipe, autosustentabilidade, próatividade e parceria com pesquisadores (modelo ETT Unicamp)

Conhecimento tácito; marketing; relacionamentos e comunicação: confiança e linguagem comum; vivências positivas em TT.

\section{Ambiente: empreendedorismo e inovação; políticas: valorização da pesquisa, PI e TTUE; informação, estrutura e equipes qualificadas; grupos de pesquisa multidisciplinares; \\ . Dificuldade em conciliar pesquisa, patenteamento e docência; barreiras informacionais e culturais; suporte administrativo/legal burocrático e centralizado.}

ETT: valorizado pelos pesquisadores; dificuldades em valorar resultados de pesquisa, negociar com as empresas, identificar/se aproximar de parceiros comerciais;

AGT facilita criação e gestão de contratos;

. Parque tecnológico: proximidade pesquisadoresindústria favorece inovação.

\footnotetext{
Criatividade, curiosidade, persistência; formação; vivências no processo aprendizagem; relacionamentos entre professores, alunos e técnicos - cooperação; relacionamentos com governo e empresas; comunicação: confiança e linguagem comum.
} 


\section{Considerações Finais}

Este estudo buscou identificar os principais intervenientes em processos de TTUE envolvendo patentes, analisando, para tanto, o caso da PUCRS. Muitos dos motivadores, obstáculos e facilitadores deste caso assemelharam-se aos identificados por outros estudos nacionais e internacionais, sendo a sistematização destes resultados de pesquisa uma das contribuições deste trabalho. Tais semelhanças justificam-se, possivelmente, pelo fato da TTUE ser um processo novo em muitos países e, assim, gerar algumas barreiras e oportunidades que se assemelham, mesmo em diferentes contextos. Além disso, a maioria dos países vem reproduzindo o modelo de TTUE adotado pelos EUA, o que pode implicar em alguns efeitos similares, tanto positivos como negativos, aplicados a realidades distintas da que o originou.

Entre os motivadores no caso da PUCRS que se destacaram dos resultados de demais estudos, estão os desafios científicos e a competição para produzir patentes. Esta competitividade foi reforçada pela percepção dos benefícios que a TTUE pode propiciar aos pesquisadores da PUCRS, sobretudo a partir do primeiro registro de patente efetuado.

O ambiente de inovação, favorecido pelo parque tecnológico e pelas políticas institucionais que valorizam a pesquisa, a PI e a TTUE, apoiando a atuação dos pesquisadores, foi ressaltado como aspecto positivo no caso da PUCRS. O acesso à informação, às estruturas de laboratórios e equipes de professores e alunos qualificados foram igualmente valorizados pelos entrevistados, assim como a atuação em grupos de pesquisa multidisciplinares, que favoreceram a geração de inventos que resultaram em patentes.

Conciliar as atividades de pesquisa e patenteamento com a atividade docente, sobretudo quando o ensino ainda se constitui como principal fonte de recursos em uma universidade privada, foi um dos desafios enfrentados pelos pesquisadores da PUCRS. Este aspecto merece especial atenção, pois o papel dos pesquisadores demonstrou ser crítico para a TTUE, embora os respondentes não tenham demonstrado ter consciência disso. Pela necessidade de desenvolver confiança, linguagem comum e transmissão de conhecimentos técnicos especializados para as empresas, os inventores da PUCRS foram essenciais na comercialização de tecnologias universitárias, aspecto corroborado por outros estudos (Guarnica \& Torkomian, 2009; Hoye \& Pries, 2009; Lipinski et al., 2008; Thursby et al., 2009).

A TTUE demanda muito envolvimento dos pesquisadores, assim, tal como propõe Silva (2007), sugere-se que as universidades criem estruturas e mecanismos institucionais que possibilitem aos pesquisadores dedicarem-se a este processo o tempo que requerido. Um programa de capacitação dos pesquisadores para desenvolver habilidades relacionais e comerciais poderia, igualmente, contribuir para o sucesso da TTUE nas universidades.

A percepção da importância da integração de conhecimentos em grupos de pesquisa multidisciplinares para a geração de inventos, no caso da PUCRS, pode estimular a constituição de grupos similares em outras universidades que buscam desenvolver a TTUE. A oportunidade de disseminação do conhecimento tácito entre universidade e empresas para a inovação propiciada pela inserção do TECNOPUC, no campus universitário da PUCRS, demonstra ser, da mesma forma, um elemento que favorece a TTUE. Portanto sugere-se que, com vistas à promoção da inovação, a existência de parques tecnológicos com participação ativa dos pesquisadores das universidades seja incentivada tanto pelas universidades como por parte das empresas e do governo.

Uma das limitações da investigação realizada, inerente a um estudo de caso único, é a impossibilidade de generalização dos seus achados de pesquisa. Para consolidar estes resultados sugere-se que o tema pesquisado seja investigado em outras universidades nacionais, sobretudo privadas, onde há maior carência de estudos empíricos. Sugere-se, ainda, a realização de estudos envolvendo outras formas de TTUE, além de patentes, bem como a investigação dos demais atores envolvidos neste processo. 
Artigo recebido em 24.03.2011. Aprovado em 21.10.2011.

\section{Referências}

Agrawal, A. (2006). Engaging the inventor: exploring licencing strategies for university inventions and the role of latent knowledge. Strategic Management Journal, 27(1), 63-79. doi: $10.1002 / \mathrm{smj} .508$

Agrawal, A., Kapur, D., \& McHale, J. (2008). How do spatial and social proximity influence knowledge flows? Journal of Urban Economics, 64(2), 258-269. doi: 10.1016/j.jue.2008.01.003

Amadei, J., \& Torkomian, A. (2009). As patentes nas universidades: análise dos depósitos das universidades públicas paulistas. Ciência da Informação, 38(2) 9-18. doi: 10.1590/S010019652009000200001

Audy, J., \& Ferreira, G. (2006). Universidade empreendedora: uma visão da PUCRS. In J. Audy \& M. Morosini (Orgs.), Inovação e empreendedorismo na universidade (pp. 417-421). Porto Alegre: EDIPUCRS.

Azagra-Caro, J., Carayol, N., \& Llerena, P. (2006). Patent production at a European research university: exploratory evidence at the laboratory level. Journal of Technology Transfer, 31(2), 257-268. doi: 10.1007/s10961-005-6110-3

Baldini, N. (2006). University patenting and licensing activity: a review of the literature. Research Evaluation, 15(3), 197-207. doi: 10.3152/147154406781775878

Baldini, N. (2010). Do royalties really foster university patenting activity? An answer from Italy. Technovation, 30(2), 109-116. doi: 10.1016/j.technovation.2009.09.007

Baldini, N., Grimaldi, R., \& Sobrero, M. (2007). To patent or no to patent? A survey of Italian inventors on motivations, incentives and obstacles to university patenting. Scientometrics, 70(2), 333-354. doi: 10.1007/s11192-007-0206-5

Bardin, L. (1977). Análise de conteúdo. Lisboa: Edições 70.

Bercovitz, J., \& Feldman, M. (2008). Academic entrepreneurs: organizational change at the individual level. Organization Science, 19(1), 69-89. doi: 10.1287/orsc.1070.0295

Bruneel, J., D'Este, P., \& Salter, A. (2010). Investigating the factors that diminish the barriers to university-industry collaboration. Research Policy, 39(7), 858-868. doi: 10.1016/j.respol.2010.03.006

Caldera, A., \& Debande, O. (2010). Performance of Spanish universities in technology transfer: an empirical analysis. Research Policy, 39(9), 1160-1173. doi: 10.1016/j.respol.2010.05.016

Castro, A., Jannuzzi, C., \& Mattos, F. (2007). Produção e disseminação de informação tecnológica: a atuação da Inova - agência de inovação da UNICAMP. Transinformação, 19(3), 265-277.

Closs, L., \& Ferreira, G. C. (2010, setembro). Transferência de tecnologia universidade-empresa: uma revisão das publicações científicas brasileiras no período 2005-2009. Anais do Encontro Nacional da Associação Nacional de Pós-Graduação e Pesquisa em Administração, Rio de Janeiro, RJ, Brasil, 34. 
Corrêa, F. (2007). A patente na universidade: contexto e perspectivas de uma política de geração de patentes na Universidade Federal Fluminense (Dissertação de mestrado). Universidade Federal Fluminense, Niterói, RJ, Brasil.

Cruz, E., \& Segatto, A. P. (2009). Processos de comunicação em cooperações tecnológicas universidade-empresa: estudos de caso em universidades federais do Paraná. Revista de Administração Contemporânea, 13(3) 430-449. doi: 10.1590/S1415-65552009000300006

Denzin, N., \& Lincoln, Y. (2005). Introduction: the discipline and practice of qualitative research. In Denzin, N., \& Lincoln, Y. (Eds.), The Sage handbook of qualitative research (pp. 1-42). Thousand Oaks: Sage.

Elfenbein, D. (2007). Publications, patents, and the market for university inventions. Journal of Economic Behavior and Organization, 63(4), 688-715. doi: 10.1016/j.jebo.2006.05.010

Freitas, H., Cunha, M., Jr., \& Moscarola, J. (1996, setembro) Pelo resgate de alguns princípios da análise de conteúdo. Anais do Encontro Nacional da Associação Nacional de Pós-Graduação e Pesquisa em Administração, Angra dos Reis, RJ, Brasil, 20.

Fujino, A., \& Stal, E. (2007). Gestão da propriedade intelectual na universidade pública brasileira: diretrizes para licenciamento e comercialização. Revista de Negócios, 12(1), 104-120.

Geoghegan W., \& Pontikakis, D. (2008). From ivory tower to factory floor? How universities are changing to meet the needs of industry. Science and Public Policy, 35(7), 462-474. doi: $10.3152 / 030234208 \times 329095$

Gil, A. (1999). Métodos e técnicas de pesquisa social (5a ed.). São Paulo: Atlas.

Golish, B. L., Besterfield-Sacre, M. E., \& Shuman, L. J. (2008). Comparing academic and corporate technology development processes. Journal of Product Innovation Management, 25(1), 47-62. doi: 10.1111/j.1540-5885.2007.00282.x

Guarnica, L., \& Torkomian, A. L. (2009). Gestão de tecnologia em universidades: uma análise do patenteamento e dos fatores de dificuldades e de apoio à transferência de tecnologia no Estado de São Paulo. Gestão \& Produção, 16(4), 624-638. doi: 10.1590/S0104-530X2009000400011

Hoye, K., \& Pries, F. (2009). 'Repeat commercializers', the 'habitual entrepeneurs' of universityindustry technology transfer. Technovation, 29(10), 682-689. doi: 10.1016/j.technovation.2009.05.008

Lei n. 10.073, de 02 de dezembro de 2004. (2004). Estabelece medidas de incentivo à inovação e à pesquisa científica e tecnológica no ambiente produtivo. Diário Oficial da União. Brasília, DF: Casa Civil.

Lester, R. (2005). Universities, innovation, and the competitiveness of local economies: summary report from the local innovation systems project - phase I [MIT IPC Working Paper 05-100]. Massachusetts Institute of Technology, Cambridge, MA.

Link, A., Siegel, D., \& Bozeman, B. (2007). An empirical analysis of the propensity of academics to engage in informal university technology transfer. Industrial and Corporate Change, 16(4), 641-655. doi: 10.1093/icc/dtm020

Lipinski, J., Minutolo, M., \& Crothers, L. (2008). The complex relationship driving technology transfer: the potential opportunities missed by universities. Institute of Behavioral and Applied Management, 9(2), 112-133.

Malhotra, N. K. (2001). Pesquisa de marketing: uma orientação aplicada (3a ed.). Porto Alegre: Bookman. 
Mariz, L. A. (2009). A criação de um setor de software entre os contextos periférico e semiperiférico: o campo organizacional como sistema aberto. Cadernos Ebape, 7(1), 1-16.

Muscio, A. (2010). What drives the university use of technology transfer offices? Evidences from Italy. Journal of Technology Transfer, 35(2), 181-202. doi: 10.1007/s10961-009-9121-7

Nonaka, I., \& Takeuchi, H. (1997). Criação de conhecimento na empresa (7a ed.). Rio de Janeiro: Campus.

Organization for the Economic Cooperation and Development. (2003). Turning science into business patenting and licensing at public research organizations. Paris: Author.

Parker, D. P., \& Zilberman, D. (1993). University technology transfers: impacts on local and U.S. economies. Contemporary Policy Issues, 11(2), 87-99. doi: 10.1111/j.14657287.1993.tb00382.x

Portaria $n^{\circ}$ 597, de 13 de setembro de 2006. (2006). Institui alterações do Estatuto da Pontifícia Universidade Católica do Rio Grande do Sul. Diário Oficial da União. Brasília, DF: Secretaria de Educação Superior do Ministério da Educação.

Póvoa, L. (2008). Patentes de universidades e institutos públicos de pesquisa e a transferência de tecnologia para empresas no Brasil (Tese de doutorado). Universidade Federal de Minas Gerais, Belo Horizonte, MG, Brasil.

Póvoa, L. M. C., \& Rapini, M. S. (2010). Technology transfer from universities and public research institutes to firms in Brazil: what is transferred and how the transfer is carried out. Science and Public Policy, 37(2), 147-159. doi: 10.3152/030234210X496619

Rapini, M., \& Righi, H. (2006). O diretório de grupos de pesquisa do CNPq e a interação universidade-empresa no Brasil em 2004. Revista Brasileira de Inovação, 5(1), 131-156.

Sampat, B. N. (2006). Patenting and US academic research in the 20th century: the world before and after Bayh-Dole. Research Policy, 35(6), 772-789. doi: 10.1016/j.respol.2006.04.009

Santana, E., \& Porto, G. (2009). E agora, o que fazer com essa tecnologia? Um estudo multicaso sobre as possibilidades de transferência de tecnologia na USP-RP. Revista de Administração Contemporânea, 13(3), 410-429. doi: 10.1590/S1415-65552009000300005

Santos, M. E., \& Solleiro, J. L. (2006). Relações universidade-empresa no Brasil: diagnóstico e perspectivas. In J. Audy \& M. Morosini (Orgs.), Inovação e Empreendedorismo na Universidade (pp. 346-376). Porto Alegre: EDIPUCRS.

Segatto-Mendes, A. P., \& Mendes, N. (2006). Cooperação tecnológica universidade-empresa para eficiência energética: um estudo de caso [Edição Especial]. Revista de Administração Contemporânea, 10, 53-75. doi: 10.1590/S1415-65552006000500004

Siegel, D., Waldman, D., Atwater, L., \& Link, A. (2004). Toward a model of effective transfer of scientific knowledge from academicians to practitioners: qualitative evidence from commercialization of university technologies. Journal of Engineering and Technology Management, 21(1-2), 115-142. doi: 10.1016/j.jengtecman.2003.12.006

Silva, E. (2007). A experiência de colaboração do departamento de engenharia metalúrgica e de materiais da UFMG com empresas - lições para a Lei de Inovação. Revista Brasileira de Inovação, 6(2), 433-459. 
Stal, E., \& Fujino, A. (2005). As relações universidade-empresa no Brasil sob a ótica da Lei de Inovação. Revista de Administração e Inovação, 2(1), 5-19. doi: 10.5585/rai.v2i1.30

Thursby, J., \& Thursby, M. (2010). Faculty participation in licensing: implications for research. Research Policy, 40(1), 20-29. doi:10.1016/j.respol.2010.09.014

Thursby, J., Fuller, A., \& Thursby, M. (2009). US faculty patenting: inside and outside the university. Research Policy, 38(1), 14-25. doi: 10.1016/j.respol.2008.09.004

Tripodi, T., Fellin, P., \& Meyer, H. (1981). Análise da pesquisa social (2a ed.). Rio de Janeiro: Editora São Francisco. 